\title{
Zivile Konfliktbearbeitung - eine vorrangige Aufgabe, aber nicht ohne Beschränkungen und Aporien
}

Frieden durch Recht mit seinem Instrument der rechtserhaltenden Gewalt beziehungsweise wie zuvor diskutiert des rechtserhaltenden Zwangs stellt eine notwendige, aber noch keine hinreichende Bedingung des Friedens dar. Bereits die EKD-Schrift von 1994 „Schritte auf dem Weg des Friedens“ verweist auf gebotene komplementäre Zugangsweisen:

„Eine internationale Friedensordnung, die bei der Aufgabe der Rechtsdurchsetzung die ultima ratio des Einsatzes militärischer Gewalt einer strengen Prüfung unterwirft und ihn tatsächlich dem Grenzfall vorbehält, ist in besonderer Weise auf den Ausbau von Wegen der zivilen Konfliktbearbeitung angewiesen.“

In der EKD-Denkschrift von 2007 stellt die zivile Konfliktbearbeitung dann auch „eine vorrangige Aufgabe“ im Rahmen des Konzeptes des gerechten Friedens dar (EKD 2007, Ziff. 170). Der Begriff der zivilen Konfliktbearbeitung ist relativ neu; er hat sich erst nach dem Ende des Ost-West-Konflikts und der Blockkonfrontation zu einem zentralen Terminus entwickelt. Friedenspolitisch verbinden sich mit der zivilen Konfliktbearbeitung drei Bedeutungen: Erstens bezieht sie sich auf den Modus des Umgangs mit gewaltsamen Konflikten und stellt insofern eine Kritik an der militärischen Vorgehensweise dar. Die Unbewaffnetheit gilt als zentraler Grundsatz ziviler Ansätze. Zweitens kommt mit dem Zivilen der Akteur in den Blick. So leisten zivile beziehungsweise zivilgesellschaftliche Akteure einen bedeutsamen friedenspolitischen Beitrag und sind ,neben staatlichen Instrumenten und Einrichtungen wichtige Träger nicht-militärischer Maßnahmen der Konfliktintervention und Vermittlung" (Weller 2007a, S. 13). Hierbei steht weniger die Abgrenzung zu staatlichen Akteuren als vielmehr die konzeptionelle Erweiterung um transnationale Kräfte im Fokus der Betrachtung (vgl. Weller 2007b, S. 69). Drittens steht zivile Konfliktbearbeitung für die „Zivilisierung 
der Konfliktbearbeitung" und damit für einen Prozess, der auf einen dauerhaften Gewaltverzicht in der Konfliktbearbeitung im Sinne der Normentwicklung und der Entwicklung einer konstruktiven Konfliktkultur zielt (Weller 2007b, S. 70). Vor diesem Hintergrund definieren Rebecca Gulowski und Christoph Weller (2017, S. 407) zivile Konfliktbearbeitung als

„die Ermöglichung sozialen Wandels durch Vergesellschaftungsprozesse, in denen die Bearbeitung von Konflikten durch Transformationen auf struktureller, institutioneller und/ oder Akteur*innen-Ebene erfolgt“".

Die Ausführungen in der Friedensdenkschrift zur zivilen Konfliktbearbeitung wie auch innerkirchliche Diskurse werden dieser Breite des Verständnisses häufig nicht gerecht. In der Regel dominiert eine Betrachtungsweise, die einseitig auf Gewaltfreiheit abhebt. In diesem Sinne konstatieren auch Andreas HeinemannGrüder und Isabella Bauer (2013, S. 20): „Zivile Konfliktbearbeitung hat sich als Gegenbegriff zu militärischen Interventionen durchgesetzt." Eine solche verengte Perspektive des Terminus birgt zumindest drei Probleme:

Zum einen besteht, wenn der Eindruck vermittelt wird, mit ziviler Konfliktbearbeitung Gewaltkonflikte generell verhindern zu können, „das Risiko von Selbstüberschätzung und Machbarkeitsillusionen" (Nachtwei 2020, S. 125). Nach Winfried Nachtwei (2020, S. 123) könne zivile Konfliktbearbeitung keine „,Globalalternative“ zu militärgestützter Sicherheitspolitik“ sein und keinen ,friedenspolitischen Alleinvertretungsanspruch“ vertreten. Diese Forderung sei insbesondere dann nicht überzeugend, wenn

- es um Fragen der staatlichen Schutzpflicht der eigenen Bürgerinnen und Bürger gegen illegale Gewalt oder von Beistandspflichten in kollektiven Sicherheitssystemen gehe,

- reale Bedrohungen und Gewaltakteure wie genozidale Gewalt, der transnationale Terrorismus oder organisierte Kriminalität kaum zur Kenntnis genommen werden,

- militärische Mittel auf der Basis von Art. 24, 25 und 87a GG unterschiedslos als „Gewaltgläubigkeit und Kriegsförderung“ angeprangert werden,

- das multilaterale und -dimensionale Krisenmanagement der letzten Jahrzehnte (von UNO, EU, NATO etc.) auf Irak, Afghanistan und Libyen reduziert werde,

- allein auf internationale Polizei gesetzt werde, ,die schon gegenüber schwer bewaffneten Milizen nicht überlebens-, geschweige durchsetzungsfähig wären“ oder 
- der Do-No-Harm-Ansatz nicht auch kritisch auf mögliche kontraproduktive Nebenwirkungen hin beleuchtet werde (vgl. Nachtwei 2020, S. 124 f.).

Zweitens fokussiert ein Verständnis ziviler Konfliktbearbeitung als Gegenbegriff militärischer Interventionen - ob bewusst oder unbewusst - auf die Konfliktphase eskalierter Gewalt. Verhindert wird damit ein notwendiger Paradigmenwechsel und eine konsequente Schwerpunktsetzung auf die Phase vor Ausbruch eines gewaltsamen Konfliktes, auf die Gewaltprävention. Dieser Shift erweist sich jedoch als zentral; so konstatiert eine groß angelegte und jüngst erschiene Studie der Vereinten Nationen und der Weltbank (2018, S. xvii):

„A shift from managing and responding to crises and toward preventing conflict sustainably, inclusively, and collectively can save lives and greatly reduce these costs.“

Die zentralen Erkenntnisse dieser Studie fasst Martin Quack (2020, S. 56 f.) in fünf Punkten zusammen. Ausgehend davon, dass Prävention zum Kernziel internationaler Kooperation werden muss, erweist sich erstens die gesellschaftliche und politische Inklusion sozialer Gruppen als wichtiger Ansatzpunkt. Nötig sind zweitens gesellschaftliche Aushandlungsprozesse. Drittens gilt es, Risiken frühzeitig und längerfristig zu adressieren. Dabei werden viertens zentrale Handlungsbereiche eruiert. Dazu gehört es, Handlungsanreize zu setzen, Institutionen inklusiv zu gestalten und strukturelle Ursachen von Benachteiligungen anzugehen. Und fünftens wird die Notwendigkeit einer kollektiven Herangehensweise betont mit dem Verantwortungsfokus auf lokale Akteure, die international unterstützt werden. Für den von dieser Studie favorisierten Shift zur Gewaltprävention sprechen sich auch Christoph Weller und Andrea Kirschner (2005, S. 24) aus. So sei zivile Konfliktbearbeitung

„kein Heilmittel für schon eskalierte Konflikte, sondern die wirksamste Maßnahme der Gewaltprävention. Die misslungene Gewaltprävention ist jedoch ein geeigneter Anlass, Lücken und Schwächen der gesellschaftlichen Institutionen für zivile Konfliktbearbeitung ausfindig zu machen sowie danach zu fragen, was die Entwicklung einer konstruktiven Konfliktkultur behindert“.

Drittens gerät die verengte Perspektive des Terminus auf Gewaltfreiheit zwangsweise in Widerspruch zu seiner dritten Bedeutung, zur Zivilisierung der Konfliktbearbeitung. Letztere hebt auf das Prozessurale des Friedens ab. Inhaltlich verbinden sich mit der Zivilisierung von Konflikten nach dem Zivilisatorischen Hexagon von Dieter Senghaas (1995) Entwicklungen wie Rechtsstaatlichkeit, 
demokratische Partizipation, Konfliktkultur, soziale Gerechtigkeit, Interdependenzen und Affektkontrolle und schließlich das Gewaltmonopol. Das heißt: Frieden als Zivilisierungsprojekt setzt auf einen funktionierenden Staat, „der ein kontrolliertes und akzeptiertes Gewaltmonopol nicht nur innehat, sondern besitzen muss“ (Gulowski und Weller 2017, S. 391). Dieser Diskurs um die inneren Spannungen zwischen gewaltfreier und zivilisierender Konfliktbearbeitung wird bislang noch zu wenig thematisiert; er muss künftig - auch innerhalb der Friedensforschung - sehr viel intensiver geführt und friedensethisch reflektiert werden.

Mit dem Ansatz der zivilen Konfliktbearbeitung verbindet sich noch eine weitere Herausforderung: Infolge ihrer unangefochten positiven Konnotation ist zivile Konfliktbearbeitung aber der Gefahr ausgesetzt, zulasten einer theoretischen Fundierung politisch instrumentalisiert zu werden (vgl. Gulowski und Weller 2017, S. 387 f.). Eine solche theoretische Auseinandersetzung und Konzeptualisierung sind aber nötig, damit „der praktische und politische Umgang mit Maßnahmen der zivilen Konfliktbearbeitung beobachtet und diskutiert werden kann" (Gulowski 2020, S. 102). Dazu bedarf es einer kritischen und differenzierten Sicht auf die Potenziale ziviler Konfliktbearbeitung: in Bezug auf die jeweiligen Konfliktphasen (Gewaltprävention, eskalierte Gewalt, Gewaltnachsorge), die verschiedenen Konfliktebenen (die strukturelle bzw. Makroebene, die institutionelle bzw. Mesoebene sowie die Akteurs- bzw. Mikroebene) sowie das Verhältnis von lokalen und internationalen Akteuren. Auf Letzteres bezieht sich Christine Schweitzer (2020, S. 82 f.), wenn sie konstatiert:

\begin{abstract}
„Das Verhältnis von lokalen zu internationalen Akteuren muss neu durchdacht werden, gibt es bei Letzteren ein weitverbreitetes Phänomen der Arroganz und Missachtung der lokalen Akteure. Die internationalen Organisationen sehen sich oft als die einzigen oder wichtigsten Akteure an und behandeln lokale Organisationen sowie lokale Aktivistinnen und Aktivisten eher als die Empfängerinnen und Empfänger von capacity building denn als Expertinnen und Experten vor Ort.“
\end{abstract}

In diesem Kontext gilt es zu reflektieren, dass auch zivile Konfliktbearbeitung von außen immer eine Form der Intervention darstellt, die das Kräftegleichgewicht vor Ort verschiebt. Vor diesem Hintergrund stellt sich hier - parallel zu militärischen Einsätzen - die Frage, ob und inwieweit internationale Akteure in der zivilen Konfliktbearbeitung ,einen konstruktiven Beitrag zu Bearbeitung der fremden Konflikte leisten (können)“" (Gulowski und Weller 2017, S. 391).

Rebecca Gulowski (2020, S. 103 ff.) spricht sich in Anlehnung an Gerechtigkeitskonzepte von Jacques Derrida und Axel Honneth für vier ethische Prinzipien ziviler Konfliktbearbeitung aus: Autonomie, Gerechtigkeit, Schadensvermeidung und Fürsorge. Die Anerkennung von Autonomie korrespondiere mit dem Prinzip 
von ownership als ,grundlegende Anforderungen an die zivile Konfliktbearbeitung von außen“ (Gulowski 2020, S. 104). Gerechtigkeitsprinzipien und damit auch Fragen nach gelingenden Anerkennungsverhältnissen regenerieren sich ,aus den Kommunikationsverhältnissen der jeweiligen Gesellschaft"; hier gelte es, „,(konflikt-)kontextsensibel immer wieder Rekonstruktionsarbeit zu leisten und zu verstehen" (Gulowski 2020, S. 104). Das Prinzip der Schadensvermeidung gehe in dem Do-No-Harm-Ansatz von Mary Anderson auf. Dabei gelte es aber auch, Spannungen zwischen verschiedenen ethischen Prinzipien mit zu berücksichtigen. Hier sieht Gulowski (2020, S. 104) ein deutliches Defizit. So werde die „Konflikthaftigkeit zwischen Schadensvermeidung und Fürsorge [...] in der zivilen Konfliktbearbeitung nicht ausreichend intensiv oder standardisiert bearbeitet" (Gulowski 2020, S. 104). Vor dem Hintergrund dieser Überlegungen entwickelt sie drei Verfahren der Wirkungsanalysen ziviler Konfliktbearbeitung:

- „Partizipation, wonach Betroffene immer in den Prozess einbezogen werden müssen,

- Rekonstruktion, das meint, dass in den Kommunikationsverhältnissen der Betroffenen mit ,ihren“ Themen die Prinzipien für deren Umgang zu generieren sind, und

- Reflexion, sprich das dialogische Nachdenken über die Wirksamkeit und Angemessenheit der Kriterien mit Blick auf die Anwendbarkeit und den Nutzen in der Praxis“ (Gulowski 2020, S. 106).

Solche Verfahren und Prüfsteine ziviler Konfliktbearbeitung können helfen, einem gerade auch in kirchlichen Kontexten vorfindlichen idealisierten Bild ziviler Konfliktbearbeitung vorzubeugen. In diesem Kontext steht beispielsweise die Kritik von Bernd Oberdorfer an den Kundgebungstext der EKD-Synode 2019 zum gerechten Frieden. So befördere der Synodentext Scheinevidenzen, wenn er einerseits von der enttäuschenden Bilanz militärischer Einsätze zur Beendigung von Menschenrechtsverletzungen spricht, andererseits aber die Grenzen ziviler Formen der Konfliktbearbeitung unerwähnt lässt. Oberdorfer (2021, i.E.) konstatiert:

„Eine selektive und zum Teil verkürzende Rezeption der Friedensforschung erzeugt in Verbindung mit einer theologisch kurzschlüssigen Ableitung der christlichen Friedensethik aus dem, gewaltlosen Friedenshandeln“ Jesu einen argumentativen Sog, der die Ablehnung militärischer Mittel des Konfliktaustrags (oder auch nur der Konfliktbegrenzung) als theologisch und, realpolitisch“ zwingend erscheinen lässt. Beides stützt sich dann wechselseitig: Was theologisch als nicht wünschenswert gilt (nämlich Friedenssicherung mit militärischen Mitteln), scheint sich ja offensichtlich auch 
,realpolitisch“ nicht bewährt zu haben; und umgekehrt bestätigen anscheinend die ,realpolitischen “ Entwicklungen den friedenstheologischen Befund.“

Auch kirchliche Akteure müssen die Aporien ziviler Konfliktbearbeitung „,Zwischen dem Wunsch nach Eindeutigkeit und Universalität sowie der Umsetzbarkeit und der Berücksichtigung partikularer Interessen und singulärer Kontexte" (Gulowski 2020, S. 89) anerkennen. Auflösen lassen sich die Spannungen zwischen Ideal und Wirklichkeit weder zugunsten der einen noch der anderen Seite. Und auch die Rolle religiöser Akteure in Konflikten und Friedensprozessen ist nicht frei von Ambivalenzen, können sie sowohl Friedensstifter als auch Brandbeschleuniger sein. Hier gilt es, näher zu qualifizieren, wie die friedensstiftenden Kompetenzen von Kirchen und Religionsgemeinschaften für die zivile Konfliktbearbeitung und friedenspolitische Praxis genutzt werden können.

Open Access Dieses Kapitel wird unter der Creative Commons Namensnennung 4.0 International Lizenz (http://creativecommons.org/licenses/by/4.0/deed.de) veröffentlicht, welche die Nutzung, Vervielfältigung, Bearbeitung, Verbreitung und Wiedergabe in jeglichem Medium und Format erlaubt, sofern Sie den/die ursprünglichen Autor(en) und die Quelle ordnungsgemäß nennen, einen Link zur Creative Commons Lizenz beifügen und angeben, ob Änderungen vorgenommen wurden.

Die in diesem Kapitel enthaltenen Bilder und sonstiges Drittmaterial unterliegen ebenfalls der genannten Creative Commons Lizenz, sofern sich aus der Abbildungslegende nichts anderes ergibt. Sofern das betreffende Material nicht unter der genannten Creative Commons Lizenz steht und die betreffende Handlung nicht nach gesetzlichen Vorschriften erlaubt ist, ist für die oben aufgeführten Weiterverwendungen des Materials die Einwilligung des jeweiligen Rechteinhabers einzuholen.

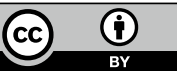

